\title{
Médiévales
}

Langues, Textes, Histoire

50 | printemps 2006

Sociétés nordiques en politique ( $\mathrm{XII}{ }^{\mathrm{e}}-\mathrm{XV}^{\mathrm{e}}$ siècles)

\section{Dominique Donadieu-Rigaut, Penser en images les ordres religieux (XII $-\mathrm{XV}^{e}$ siècles)}

Paris, Éditions Arguments, 2005, 385 p., 111 ill. (préface de Jean-Claude Schmitt).

Jérôme Baschet

\section{(2) OpenEdition}

1 Journals

Édition électronique

URL : https://journals.openedition.org/medievales/1412

DOI : $10.4000 /$ medievales. 1412

ISSN : 1777-5892

Éditeur

Presses universitaires de Vincennes

\section{Édition imprimée}

Date de publication : 1 juin 2006

Pagination : 180-182

ISBN : 2-84292-186-0

ISSN : 0751-2708

Référence électronique

Jérôme Baschet, " Dominique Donadieu-Rigaut, Penser en images les ordres religieux ( $x \|^{e}-x v^{e}$ siècles) 》, Médiévales [En ligne], 50 I printemps 2006, mis en ligne le 09 février 2007, consulté le 22 avril 2022.

URL : http://journals.openedition.org/medievales/1412; DOI : https://doi.org/10.4000/medievales.

1412

Ce document a été généré automatiquement le 22 avril 2022.

Tous droits réservés 


\section{Dominique Donadieu-Rigaut, Penser en images les ordres religieux ( $\mathrm{XII}^{e}$ - $X V^{e}$ siècles)}

Paris, Éditions Arguments, 2005, 385 p., 111 ill. (préface de Jean-Claude Schmitt).

Jérôme Baschet

1 Le livre de Dominique Donadieu-Rigaut est une belle réussite et il faut féliciter d'emblée l'auteure et son éditeur pour le soin apporté à ce volume et la qualité du dossier iconographique : 111 illustrations, presque toutes en couleur (mais parfois de format trop réduit), c'est un véritable exploit ! Clairement défini, l'objet du livre est d'analyser les modalités d'auto-représentation des différents ordres religieux, non tant par une accumulation de symboles identitaires propres à chacun d'eux, mais plutôt en cernant la manière qu'ils ont de se penser comme ordo, comme communauté spécifiquement ordonnée au sein de l'Église universelle. Soulignons d'emblée deux mérites de ce travail. Le premier est de ne pas s'en tenir à une approche monographique, centrée sur un ordre spécifique, mais d'adopter une perspective comparatiste, prenant en compte sinon tous les ordres médiévaux, du moins un nombre important d'entre eux. Cela est pleinement justifié et nécessaire, dès lors que l'identité de chaque ordre est relative, jouant à la fois d'une différenciation avec ses concurrents et d'emprunts nombreux, faisant circuler des modèles d'un ordre à l'autre. En second lieu, fondant son enquête sur les images (sans négliger la nécessité de bien connaître les textes et l'histoire propres à chaque ordre), Dominique Donadieu-Rigaut s'appuie résolument sur le concept de pensée figurative, emprunté à Pierre Francastel. Loin d'être un simple postulat, cette référence féconde se manifeste par une extrême attention aux modes de fonctionnement des œuvres visuelles, c'est-à-dire à des aspects que certains diraient purement « formels ", mais qui sont la matière même d'une pensée en images : couleurs et tonalités, contacts, superpositions, symétries, lignes (comme cette "courbe qui parle »), voilà bien ce qui permet de créer visuellement des relations, des liens, des oppositions, des hiérarchies, c'est-à-dire de mettre l'ordre en image. Il en résulte de très 
belles analyses d'images, attentives et subtiles, toujours liées à une problématisation historique, de surcroît rédigées sans pesanteur mais non sans d'heureuses formules.

2 L'originalité du livre se dégage ainsi : il ne traite ni d'un seul ordre religieux, ni d'un seul thème iconographique, mais croise une multiplicité de thèmes et d'institutions. $\mathrm{Ce}$ foisonnant réseau (un « hyperthème ») est le véritable objet d'une recherche qui avoue avec pertinence s'intéresser tout particulièrement à "ce qui se joue entre les images ", c'est-à-dire aux échos, aux reprises, aux modalités de circulation et de transformations des thèmes et des motifs, lors de leurs migrations entre plusieurs ordres. En ce point, et tout en comprenant le souhait d'alléger l'édition de l'ouvrage, on suggèrera que, dans le cadre d'une démarche qui se revendique de la sérialité, il aurait été bon d'évoquer, par exemple en annexe, les corpus systématiques dont ces œuvres font partie. Par ailleurs, si le choix de thèmes dont la particularité est de circuler entre les ordres est parfaitement justifié, quelques allusions à d'autres séries, comme les grands cycles d'auto-représentation (notamment dominicains), auraient peut-être permis d'enrichir encore, par comparaison, les analyses proposées ici. Mais, si le lecteur peut ressentir ici un manque, ce sont là deux aspects que l'auteure n'ignore nullement et qu'elle n'a pas manqué de prendre en compte dans sa recherche.

3 Après un premier chapitre consacré aux Bibles moralisées, qui permet de situer les ordres religieux au sein des articulations fondamentales de la société médiévale (clercs/laïcs, séculiers/réguliers, ordres traditionnels/Mendiants), cinq thèmes sont successivement examinés : les Vierges au manteau couvrant un ordre religieux, la prise $\mathrm{d}$ 'habit (essentiellement du fondateur), le don de la règle, la mort du fondateur, les arbres monastiques. Hormis le premier, tous ces thèmes mettent l'accent sur la relation entre l'ordre et son fondateur, aspect qui constitue certainement le principal fil directeur de la recherche. La Vierge au manteau fait donc exception, dans la mesure où la communauté fraternelle de l'ordre y est exprimée par son rapport non au père fondateur mais à Marie, selon un modèle également appliqué à la totalité de la société chrétienne. L'auteure fait l'hypothèse que ce thème est adopté par des ordres dont le fondateur est une figure plutôt faible, ou du moins dédoublée (les Chartreux et, dans une moindre mesure, les Cisterciens) : c'est lorsque le Père défaille que la Mère s'offre comme recours pour manifester l'unité de la communauté de l'ordre. Il est vrai que les ordres dont les fondateurs constituent des modèles absolus (Bénédictins et Franciscains) n'ont pas recours à cette image. Mais on pourra exprimer quelques doutes tant il y a, dans les schèmes chrétiens, une capacité à conjoindre et à combiner - plutôt qu'à opposer - paternité (des clercs, par exemple) et maternité (de l'Église, par exemple). Quoi qu'il en soit, la reprise de cette image chez les Dominicains, par l'effet d'une concurrence explicitement assumée, permet de montrer que l'opposition entre ordres traditionnels et Mendiants ne constitue nullement une frontière infranchissable pour l'iconographie. Un autre exemple de ce processus, également bien analysé, concerne les représentations de la mort de François ou de Dominique, qui transforment celles de la mort de Benoît, d'une manière qui s'éloigne de la tradition textuelle mais qui permet de mieux exprimer la communauté des disciples créée autour du corps du fondateur.

4 Parmi les autres thèmes étudiés, le don de la règle est l'un des principaux. Il est riche de variantes, qui vont au-delà de l'opposition principale entre les ordres traditionnels, auxquels le fondateur donne lui-même la règle (ainsi, Benoît multipliant ses fils spirituels par la diffusion de sa règle), et les Mendiants, dont l'approbation de la forme 
de vie relève de l'autorité pontificale (avec cette spécificité des Franciscains, qui font des stigmates le sceau véritable par lequel le Christ lui-même vient confirmer la règle). Enfin, les arbres monastiques, à partir des années 1430, font l'objet d'un passionnant dossier, associant Bénédictins, Chartreux, Dominicains et Franciscains. Ces arbres, dont le fondateur, tel Jessé, est la racine et les disciples les fruits, ne représentent évidemment pas des généalogies mais des "fraternités ", exprimant la fécondité spirituelle du fondateur. Soulignant qu'il serait vain de vouloir y repérer quelque succession chronologique que ce soit, l'auteure met en évidence différents modes de structuration, souvent finement élaborés, jouant parfois de strictes hiérarchies pour signifier la dilatation et le succès de l'ordre. Elle décèle dans certains exemplaires une véritable structure mémorielle, associant une géographie sélective et auto-centrée et une combinatoire de temporalités spécifiquement signifiantes. Surtout, Dominique Donadieu-Rigaut fait voir avec talent quels écarts se jouent entre les arbres bénédictins, dominicains et franciscains. Tandis que l'arbre-ordo dominicain s'identifie à l'arbre de Jessé au point d'y intégrer également la Vierge à l'enfant, l'arbre-ordo franciscain, inspiré par l'iconographie du Lignum vitae, fusionne avec la croix du Christ. Outre qu'elle permet de rappeler l'identification de François au Christ, cette dernière option présente la particularité de ne pas situer le fondateur à la racine de son ordre : il cède la place aux vertus dont il est lui-même le serviteur et adopte une position qui est moins celle d'un père fondateur que d'un frère au milieu de ses disciples.

Le réseau de thèmes étudié par Dominique Donadieu-Rigaut met l'accent sur la représentation de l'ordre comme communauté régie par les règles de la parenté spirituelle. L'unité des frères s'y articule, de manière plus ou moins marquée selon les cas, à leur rapport commun au père fondateur (ou par exception à la Vierge). À cet égard, les images étudiées jouent singulièrement de la temporalité : elles cherchent, notamment dans les milieux de l'Observance, à court-circuiter la distance avec le temps des origines pour manifester l'éternelle présence du fondateur dans la vie de son ordre. Par ailleurs, dans la mesure où la parenté spirituelle sert également de modèle pour penser la société chrétienne dans son ensemble, la représentation des ordres religieux pose la question de l'articulation entre une communauté spécifique et l'Ecclesia dont elle fait partie, bien qu'elle soit parfois tentée de la signifier dans sa totalité. Enfin, l'auto-représentation des ordres comporte une forte dimension relative, différentielle, qui va au-delà des oppositions entre ordres traditionnels et Mendiants, ou entre tel ordre faisant de la figure du fondateur le cœur de son identité, comme les Mineurs, et tel autre qui construit ses références de manière plus collective, comme les Prêcheurs. De telles oppositions sont en partie pertinentes, mais le réseau de thèmes construit par Dominique Donadieu-Rigaut invite à une lecture plus fine, attentive aux circulations en tous sens des thèmes et des motifs, à la complexité des emprunts et des réinterprétations. Son beau livre montre avec quelle force et avec quelles ressources la pensée figurative participe à ce travail permanent d'auto-représentation, combinant intégration et différenciation, par lequel chaque ordre religieux pense sa propre cohésion, tout en s'inscrivant dans l'Unité diversifiée de la chrétienté. 